\title{
The Effects of Timing Jitter in Sampling Systems
}

\author{
T. MICHAEL SOUDERS, MEMBER, IEEE, DONALD R. FLACH, MEMBER, IEEe, CHARLES HAGWOOD, \\ AND GRACE L. YANG
}

\begin{abstract}
Timing jitter generally causes a bias (systematic error) in the amplitude estimates of sampled waveforms. Equations are developed for computing the bias in both the time and frequency domains. Two principal estimators are considered: the sample mean and the socalled Markov estimator used in some equivalent-time sampling systems. Examples are given using both real and simulated data.
\end{abstract}

\section{INTRODUCTION}

$\mathrm{T}$ HE PURPOSE of waveform sampling and digitizing systems is to assign an amplitude value and a corresponding time of occurrence to each of a finite set of discrete points that define a waveform over a specified time epoch. In this paper we present an analysis of the effects of random errors in the time of occurrence of the waveform samples. Such errors are commonly called timing jitter. Since only the nominal sample times are known, the effect of timing jitter is to introduce random errors in the amplitude values which are assumed to correspond to the sample times. In general, estimates of the true waveform values that are based on the observed samples tend to be biased. This is generally true for any sampled waveform for which second and higher order derivatives exist. The bias depends on the statistical estimator that is used. We have examined estimators used in both real-time and equivalent-time sampling processes.

While it is true that waveform estimates based on timejittered samples are generally biased, the power, or mean squared value of the waveform samples is asymptotically unbiased, provided that the jitter is uncorrelated with the waveform itself. Therefore, it will be shown that the power contained in the noise created by timing jitter is directly related to the bias described above.

In recent years, timing jitter has been studied either directly [1] or indirectly [2], [3] by several authors. In this work, we expand on the results using the mean in equivalent-time sampling given by Gans [1], and include analyses of other estimation processes commonly used in equivalent-time sampling.

\section{Problem}

Referring to Fig. 1, consider a waveform to be sampled, described by $y=f(t)$. For jitter-free sampling, we

Manuscript received April 26, 1989.

T. M. Souders, D. R. Flach, and C. Hagwood are with the National Institute of Standards and Technology, Gaithersburg. MD 20899.

G. L. Yang is with the Department of Mathematics. University of Maryland, College Park, MD 20740.

IEEE Log Number 8931817.

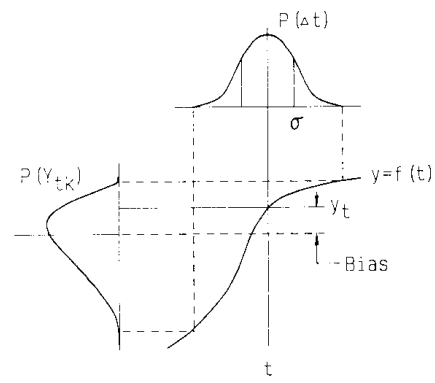

Fig. I. Illustration of the bias associated with using the mean to estimate the value of a waveform sampled at time $t$, with timing jitter having a PDF of $P(\Delta t)$.

write $y_{t}=f(t)$, where $y_{t}$ is the amplitude at time $t$. With jitter present, let $\left[y_{t 1}, \cdots y_{t n}\right]$ be the statistical sample, where $y_{t k}=f\left(t+\Delta t_{k}\right)$ and $\Delta t_{k}$ is a random variable denoting the $k$ th deviation from the nominal sampling time, $t$. (For $n>1$, multiple sampling at the same nominal time $t$ on the waveform is assumed, as in equivalenttime sampling.) We wish to determine the bias that results when using an estimator $\hat{y}_{t}$, based on the statistical sample $\left[y_{t 1}, \cdots y_{t n}\right]$.

\section{The Mean as Estimator}

For real-time sampling, the most commonly used estimator is the sample mean

$$
\bar{Y}_{t}=\frac{1}{n} \sum_{k=1}^{n} y_{t k}
$$

which, in the limit as $n$ becomes large, approaches the expectation of $Y_{t}$, where $Y_{t}$ denotes any one of the $y_{t k}$ in the statistical sample. This is given by

$$
E\left[Y_{t}\right]=\int_{-\infty}^{\infty} f(t+\Delta t) P(\Delta t) d \Delta t
$$

where $P(\Delta t)$ is the probability density function (PDF) of the jitter distribution, as illustrated in Fig. 1.

For the more usual case of additive random noise in the measured signal, the mean is an unbiased estimator. Therefore, when it is possible to take repeated measurements, the sample mean is frequently used to reduce noise by averaging repeated data records. In other cases, single records are often processed using orthogonal transformations or least-squares fitting routines to extract signals from noise. Fourier transforms and sine wave curve fitting, for example, are commonly used [4], [5]. For these 
cases, the mean is the underlying estimator; the resulting coefficients approach the coefficients of the transform of $E\left[Y_{t}\right]$, as the number of samples increases.

When timing jitter is present in addition to additive noise, a bias will be introduced in the cases noted above. The bias can be calculated in terms of the moments of the jitter distribution by expanding $f(t+\Delta t)$ in (2) in a Taylor series:

$$
\begin{aligned}
E\left[Y_{t}\right]= & \int_{-\infty}^{\infty}\left[f(t)+f^{\prime}(t) \Delta t\right. \\
& \left.+f^{\prime \prime}(t) \Delta t^{2} / 2 !+\cdots\right] P(\Delta t) d \Delta t
\end{aligned}
$$

where (') represents the derivative. Since the integral of the first product term in (3) is the true value $f(t)$, the remaining terms of (3) comprise a bias. The bias is actually the sum of products of the function's derivatives and the moments of the underlying jitter distribution. We can express this result in a more useful form in the frequency domain by taking the Fourier transform of (3):

$$
\begin{aligned}
& F\left(E\left[Y_{t}\right]\right) \\
& =F\left[f(t) \int_{-\infty}^{\infty} P(\Delta t) d \Delta t\right] \\
& +F\left[f^{\prime}(t) \int_{-\infty}^{\infty} \Delta t P(\Delta t) d \Delta t\right] \\
& +F\left[f^{\prime \prime}(t) \frac{1}{2} \int_{-\infty}^{\infty} \Delta t^{2} P(\Delta t)^{2} d \Delta t\right]+\cdots
\end{aligned}
$$

The integrals in (4) are, respectively, the following constants: unity, the mean $(\mu)$, and the second moment $\left(\sigma^{2}\right.$ $\left.+\mu^{2}\right)$, of the jitter distribution. Since $F\left(b \cdot f^{\prime}(t)\right)=$ $j \omega b \cdot F(f(t))=j \omega b \cdot H(\omega),(4)$ becomes

$$
\begin{aligned}
F\left(E\left[Y_{t}\right]\right)= & H(\omega)+j \omega \mu H(\omega) \\
& -\omega^{2}\left(\sigma^{2}+\mu^{2}\right) H(\omega) / 2+\cdots \\
= & H(\omega)\left[1+j \omega \mu-\omega^{2}\left(\sigma^{2}+\mu^{2}\right) / 2\right. \\
& +\cdots] .
\end{aligned}
$$

The bracketed terms of (6) represent a filtering function operating on the true signal $H(\omega)$. The second term within the brackets indicates the intuitively obvious notion that a nonzero mean in the jitter distribution will show up as an apparent linear phase shift in the frequency domain. (The corresponding term in (3) shows a fixed time shift.) Higher order terms involve the higher order moments of the jitter distribution; for symmetrical distributions, the odd-order terms vanish.

Note that if either $f(t)$ or $P(\Delta t)$ in (2) is an even function, then (2) becomes equivalent to a convolution integral. In this case, the filter function can be calculated in closed form as the Fourier transform of the jitter PDF. Using this approach, the exact filter functions for Gauss-

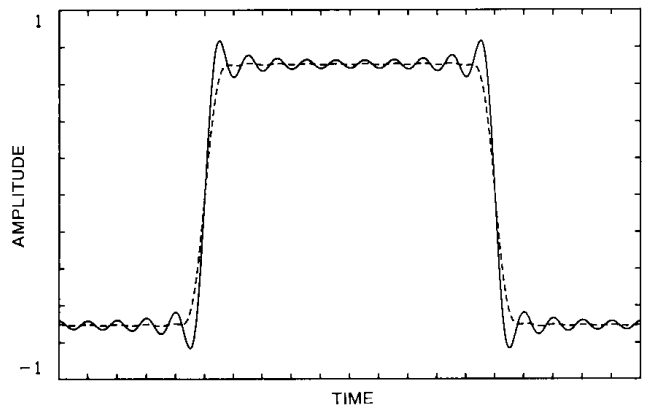

(a)

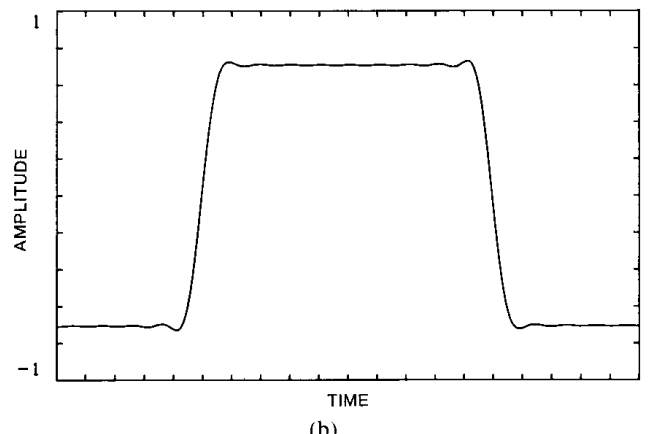

Fig. 2. The filtering effects of averaging repeated, time-jittered records (a) The solid curve consists of the first ten harmonic components of a square wave; the dashed curve gives the computed. filtered waveform. based on uniform jitter with a standard deviation of one tenth the square wave period. (b) The mean of 100 time-skewed records of the example waveform is given. The time skew had the same standard deviation used in (a)

ian and uniform distributions (with $\mu=0$ ) have been calculated to be $e^{-\omega^{2} \sigma^{2} / 2}$ and sinc $(\sqrt{3} \omega \sigma)$, respectively.

In many applications where the jitter distribution spans a time window that is short compared to the period of the highest frequency component of the signal, the three terms shown in (6) will be an adequate approximation. Then, only the mean and the second moment of the distribution need be known to predict and compensate for the errors. For example, with a Gaussian distribution having a $\sigma$ of one tenth the signal period $2 \pi / \omega$, the higher order terms amount to only 1.8 percent out of a total bias of 17.9 percent. For a uniform distribution having the same ratio of $\sigma$ to signal period, the respective values are 1.1 percent out of 18.6 percent

\section{A. Examples}

The results expressed in (6) can be used in a variety of situations. The most obvious are cases in which multiple samples of the same nominal time point on a waveform are averaged. This occurs in equivalent-time sampling systems such as sampling oscilloscopes, in so-called random repetitive sampling in which the time of occurrence of random samples is quantized, and in cases where multiple records of the same waveform are averaged, but the records have random time-skew up to one sample period between them due to asynchronous triggering. In the latter two examples, the jitter distribution is uniform, and 


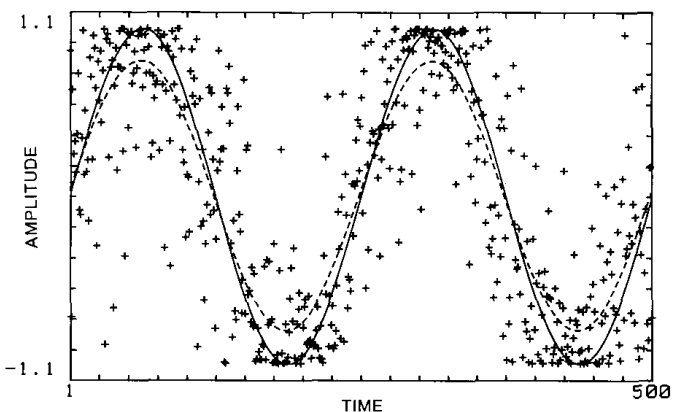

Fig. 3. Results from sine wave curve fitting of time-jittered data, with a jitter standard deviation of $36^{\circ}$. The solid curve is the unjittered waveform which has a peak value of 1.0 , and the jittered samples are represented by $(+)$. The fitted waveform is given by the dashed curve, and has a peak value of 0.804

has a known variance based on the digitizing resolution in the first case or the sampling period in the second.

Fig. 2 shows the results of averaging multiple records simulated with random time-skew between them. The waveform is the sum of the first 10 harmonic components of a square wave, and the time-skew distribution is uniform, with a width of 5.6 percent of the square wave period. Fig. 2(a) shows the original waveform as the solid curve, and the dashed curve is the expected waveform after filtering with the function given in (6). In Fig. 2(b), the waveform shown after averaging 100 time-skewed records is very close to the expected waveform.

In Fig. 3, the results of a sine wave curve fit are shown for simulated data: a sine wave of unit peak amplitude sampled at 500 points, under the influence of Gaussian jitter with $\sigma=36^{\circ}$. The solid curve is the original waveform, and the plotted points are the time-jittered samples. The dashed curve is a plot of the fitted sine wave, obtained using the three-parameter fit described in [6]; the peak amplitude of the fitted sine wave, 0.804 , is also indicated. The attenuation predicted by (6) gives a peak amplitude of 0.821 . The rms value of the residuals in this case is 0.409 .

\section{The Median and Markov Estimators}

In some equivalent-time sampling systems, the estimator is known only in algorithmic rather than analytic form. The sampling voltage tracker [7] is a good example. In this approach, shown schematically in Fig. 4, the waveform is sampled repeatedly at the same nominal point, $t$, on the waveform with a strobed analog comparator. The samples, $y_{t k}$, are compared with a reference input, $y_{r}(t)$ which is incremented or decremented by a small, fixed amount, $\delta$, each period, depending on the logical output of the previous comparison. Under these conditions, the reference voltage follows a random walk described by a Markov chain. Fig. 5 illustrates this process. The estimate of $y_{t}$ is obtained by integrating the reference voltage over a large number of repetitions and digitizing with a DVM. Therefore, at each $t$ the sample mean

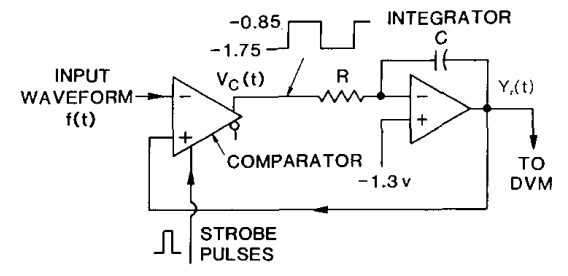

Fig. 4. Basic circuit of the sampling voltage tracker.

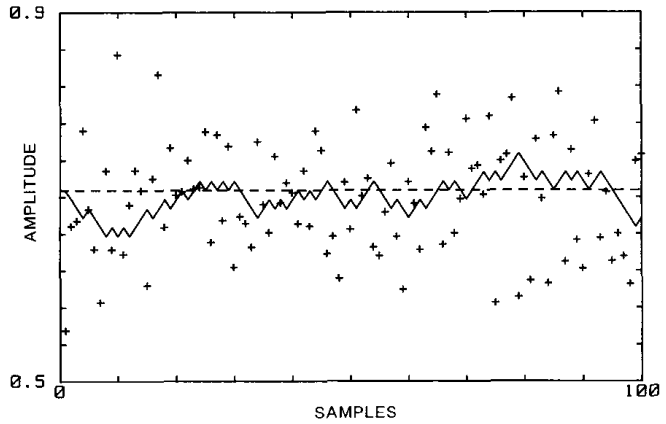

Fig. 5. Illustration of Markov process, representative of the output wave form $Y_{r}(t)$ in Fig. 4. The solid curve is the Markov chain response to the samples designated by $(+)$. The samples are taken from a sine wave at $45^{\circ}$, with Gaussian jitter with $\sigma=36^{\circ}$.

of the Markov chain is the estimator for the true value, $y_{i}$; the individual data are processed in real-time and are not stored.

The statistics of this so-called Markov estimator have been studied by the authors [8]. The analysis is too lengthy to include here; however, the results are clear: the Markov estimator asymptotically converges to the population median of $Y_{t}$ as $n \rightarrow \infty$ and then $\delta \rightarrow 0$. An intuitive appreciation of why this may be so follows from the observation that, for $\delta \ll|a|$, where $a=f(t+\Delta t)-$ $y_{r}(t)$, the decision to increment or decrement is dependent only on the sign of $a$. Therefore, the mean of $y_{r}(t)$ will be stable only when the sign of $a$ has equal probability of being positive or negative. This is the condition for which the mean of $y_{r}(t)$ is the median of the samples $y_{t k}$.

Although a formal proof has not yet been found, a similar argument suggests that a modification of the algorithm described above could be used to find any given percentile of a sample set. To accomplish this, simply set the ratio of increment amount, $\delta \uparrow$, to decrement amount, $\delta \downarrow$, equal to $p \div(1-p)$, where $p$ is the percentile desired.

As an estimator, the Markov estimator has some unique properties relevant to the problem of time-jittered sampling. For symmetrical jitter distributions with zero mean, the median of the time-jittered sample set $\left[v_{t 1}, \cdots v_{t n}\right]$ is unbiased, for monotonic $f(t)$ [9]. Furthermore, for distributions with nonzero median, it is easily seen that the bias is simply a constant time shift, as in the case of the sample mean. (If the distribution has a median, $m$, then the distribution of $(\Delta t-m)$ will have a median of zero.) 
Another unique feature of the Markov estimator is its computational efficiency. In usual sorting algorithms the time needed to compute the median is of the order $n \log$ $(n)$. An algorithm to implement the Markov estimator is of order $n$; hence, its cost is of the same order as that required to compute the mean. Furthermore, the memory requirements are equally minimal for real-time operation: only the running average must be stored.

For nonmonotonic signals, the bias associated with the median is not generally known; however, it can be computed under certain conditions. For example, for symmetrical jitter distributions and signals that are symmetric about their maxima or minima, $t_{m}$, the bias is given by $f\left(t_{m}+\Delta t_{m}\right)-f\left(t_{m}\right)$, where $\Delta t_{m}$ is the one-sided median of the jitter distribution, as illustrated in Fig. 6. When the signal is a sine wave, for example, the respective biases for Gaussian and uniform jitter distributions (with $\sigma=$ $10^{\circ}$ ) are 0.70 and 1.14 percent. When the mean is the estimator, the respective biases are 1.52 percent in both cases.

For monotonic regions of repetitive signals, the median (or Markov estimator) is obviously the best choice for an estimator, since it is asymptotically unbiased. Few signals of interest, however, are strictly monotonic. Nevertheless, the median may still be the best estimator for some applications in which the signals are not monotonic. As indicated above, for sine waves sampled with either Gaussian or uniformly distributed jitter, the median gives a smaller bias than the mean for all values of the independent variable. As another example, consider the measurement of pulse rise time. Provided that the pulse has a monotonic transition over the designated region, the median will give unbiased estimates of the defining 10- and 90 -percent points, and the rise time measurement will be unbiased. This will not be the case if the mean is used.

\section{A. Examples}

In Fig. 7, a comparison is made between the Markov estimator and the sample mean estimator, for a sine wave sampled with Gaussian jitter with $\sigma=10^{\circ}$. At each $t$, 360000 observations are simulated. Fig. 7(a) is a plot of $\sin (t)$, Fig. $7(\mathrm{~b})$ is a plot of the bias of the sample mean estimator, and Fig. 7(c) is a plot of the bias of the Markov estimator for $\delta=10^{-4}$. Note that, as predicted, the Markov estimator shows no bias in the monotonic regions of the waveform, and that its bias is smaller everywhere than that of the sample mean.

Fig. 8 gives the results of data taken with the sampling voltage tracker. In this example, a filtered, high-purity $10-\mathrm{MHz}$ sine wave has been sampled using the system illustrated in Fig. 4 and described in [7]. Jitter was created by phase-modulating the sine wave with Gaussian noise. A sine fit was performed on the samples, and the residuals are plotted in the figure. The fundamental sine wave component seen in the residuals is an artifact of the fitting process: the expected bias (see Fig. 7) has a sine wave component itself, which has been removed by the curve fitting process to minimize the squared error.

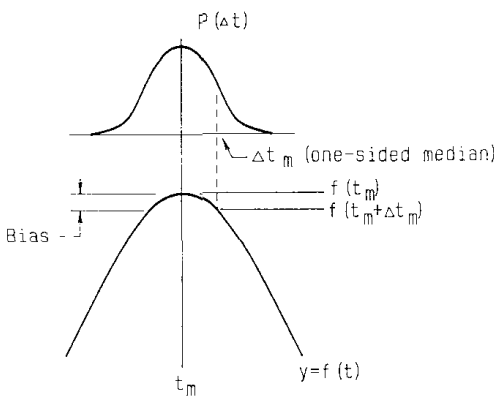

Fig. 6. Illustration of the bias associated with the median estimator, for symmetrical jitter distributions, and nonmonotonic waveforms which are symmetrical about their maxima or minima.

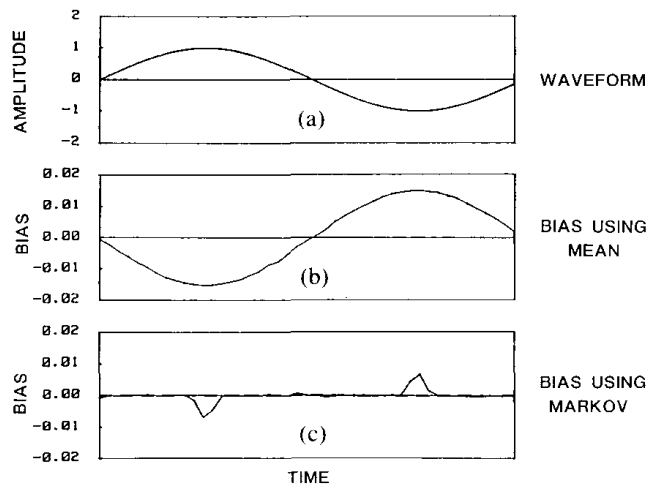

Fig. 7. Simulation results comparing the Markov and sample mean esti mators, for a sine wave sampled with Gaussian jitter with $\sigma=10^{\circ}$ and $n=360000$. (a) The original waveform. (b) The bias using the sample mean. (c) The bias using the Markov estimator is given for $\delta=10^{-4}$.

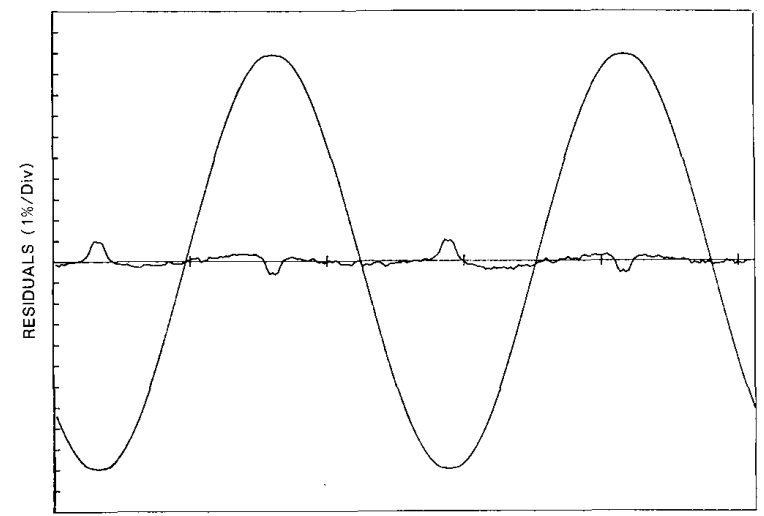

TIME (40ns/Div)

Fig. 8. Actual SVT results based on sampling a sine wave with added jitter. A plot is shown of the residuals from a sine wave curve fit, with the original sine wave overlaid for reference. The vertical scale applies to the residuals; the original waveform scale is 10 times larger

\section{Signal Power and Curve Fit Residuals}

As stated previously, the power (or mean squared value) of the time-jittered samples of a periodic signal is independent of the jitter for random jitter that is uncorrelated 
with the signal. Thus for an arbitrary, periodic function, $z=G(t)$, defined over the range $t=\left[t_{0}, t_{n-1}\right]$, having Fourier coefficients $a_{1} / \sqrt{ } 2, \cdots a_{m} / \sqrt{ } 2$, Parseval's relation gives

$$
\frac{1}{n} \sum_{t=t_{0}}^{t_{n-1}} E\left[G^{2}(t+\Delta t)\right]=\sum_{k=1}^{m} a_{k}^{2} / 2
$$

the square root of which is the rms value of $z$, the function without time-jitter. A proof of (7) is given in the Appendix.

From (6), we know the Fourier coefficients of $E[G(t$ $+\Delta t)$ ], i.e., the expectation of the time-jittered version of $z$, are given by

$$
F(E[G(t+\Delta t)])=\frac{a_{k}}{\sqrt{2}}\left(1-\frac{\omega_{k}^{2} \sigma^{2}}{2} \cdots\right) .
$$

In the limit for $m$ finite and $t_{n} \rightarrow \infty$, the $m$ expected Fourier coefficients of $G(t+\Delta t)$ are given by (8), and the remaining energy will be represented by additional noise spectral components, the total power of which we call $a_{j}^{2}$. Since we have shown that the total signal power is

$$
\sum_{k=1}^{m} \frac{a_{k}^{2}}{2}=\sum_{k=1}^{m} \frac{a_{k}^{2}}{2}\left[1-\frac{\omega_{k}^{2} \sigma^{2}}{2}\right]^{2}+a_{j}^{2}
$$

it follows that

$$
a_{j}^{2} \simeq \sum_{k=1}^{m} \frac{a_{k}^{2} \omega_{k}^{2} \sigma^{2}}{2} .
$$

For sine fitting, the power in the residuals equals the power lost due to the bias of the fitted function. (To avoid truncation errors, this applies only to sine waves that have been sampled over an integral number of cycles.) In Fig. 3 , for example, the calculated mean-squared value of the residuals was 0.170 , compared with 0.176 , the computed difference between the power of the original, unjittered waveform, and the power represented by the fitted sine wave. For Fourier transforms of periodic functions sampled over an integral number of periods, the power lost due to the bias of the "true" harmonic components is spread out in additional noise components.

\section{Conclusions}

In general, waveform estimates that are based on samples taken with timing jitter tends to be biased. We have derived expressions for the bias that results from using the sample mean as an estimator, and have shown that it can be approximated in the frequency domain by a simple filter function. The so-called Markov estimator used in the sampling voltage tracker, for example, was also studied, and was shown to asymptotically converge to the population median. It is, therefore, an unbiased estimator for monotonic waveforms sampled with jitter distributions having a median of zero.

While the Markov estimator is expected to give a smaller bias than the mean in many practical cases, it should be borne in mind that the filter function associated with the mean is a linear operator, while the Markov es- timator is not. This means that the bias in the former case is more easily compensated for, as pointed out by Gans [1].

\section{APPENDIX}

We want to show that

$$
\frac{1}{n} \sum_{t=t_{0}}^{t_{n}-1} E\left[G^{2}(t+\Delta t)\right]=\sum_{k=1}^{m} a_{k}^{2} / 2
$$

where $G(t)$ is an arbitrary periodic function having (real and imaginary) Fourier coefficients $a_{1} / \sqrt{ } 2, \cdots a_{m} / \sqrt{ } 2$, sampled over one period defined by the range of discrete values $t=\left[t_{0}, t_{n-1}\right]$, where $n$ satisfies the sampling theorem. We will prove (A1) for $G(t)=a_{1} \cdot \sin ($ it $)$ and $G(t)=a_{2} \cdot \cos (i t)$, where $i$ is $2 \pi$ times an integer; it will then follow from the law of superposition for expectations of random variables that (A1) holds for any $G(t)$ which can be defined by a Fourier series.

By expanding $\sin (i t+i \Delta t)$ using the trigonometric identity, we have

$$
\begin{aligned}
E\left[a^{2} \cdot\right. & \left.\sin ^{2}(i t+i \Delta t)\right] \\
= & a^{2} \cdot \sin ^{2}(i t) \int_{-\infty}^{\infty} \cos ^{2}(i \Delta t) \cdot P(\Delta t) d(\Delta t) \\
& +2 a^{2} \cdot \sin (i t) \cos (i t) \int_{-\infty}^{\infty} \sin (i \Delta t) \cos (i \Delta t) \\
& \cdot P(\Delta t) d(\Delta t) \\
& +a^{2} \cdot \cos ^{2}(i t) \int_{-\infty}^{\infty} \sin ^{2}(i \Delta t) \cdot P(\Delta t) d(\Delta t) \\
= & a^{2} \cdot \sin ^{2}(i t) \int_{-\infty}^{\infty} P(\Delta t) d(\Delta t) \\
& -a^{2}\left[\sin ^{2}(i t)-\cos ^{2}(i t)\right] \int_{-\infty}^{\infty} \sin ^{2}(i \Delta t) \\
& \cdot P(\Delta t) d(\Delta t) \\
& +2 a^{2} \cdot \sin (i t) \cos ^{\infty}(i t) \int_{-\infty}^{\infty} \sin ^{\infty}(i \Delta t) \cos (i \Delta t) \\
& \cdot P(\Delta t) d(\Delta t) .
\end{aligned}
$$

Since

$$
\begin{gathered}
\int_{-\infty}^{\infty} P(\Delta t) d(\Delta t)=1 \\
\sum_{t=t_{0}}^{t_{n-1}} a^{2}\left[\sin ^{2}(i t)-\cos ^{2}(i t)\right]=0
\end{gathered}
$$

and

$$
\sum_{t=t_{0}}^{t_{n-1}} 2 a^{2} \cdot \sin (i t) \cos (i t)=0
$$


it follows that

$$
\begin{aligned}
& \frac{1}{n} \sum_{t=t_{0}}^{t^{\prime \prime}} E\left[a^{2} \cdot \sin ^{2}(i t+i \Delta t)\right] \\
& \quad=\frac{1}{n} \sum_{t=t_{0}}^{t_{n}} a^{2} \cdot \sin ^{2}(i t)=a^{2} / 2 .
\end{aligned}
$$

An equivalent derivation exists for $G(t)=a \cdot \cos (i t)$.

\section{REFERENCES}

[1] W. L. Gans, "The measurement and deconvolution of time jitter in equivalent-time waveform samplers," IEEE Trans. Instrum. Meas., vol. IM-32, Mar. 1983
[2] B. Liu and T. P. Stanley, "Error bounds for jittered sampling," IEEE Trans. Automat. Contr, vol. AC-10. Oet. 1965.

[3] Y. C. Jenq, "Digital spectra of non-uniformly sampled signals: fundamentals and high-speed waveform digitizers, "IEEE Trans. Instrum. Meas., vol. 37, June 1988.

[4] B. E. Peetz, "Dynamic testing of waveform recoriers," IEEE Trans. Instrum. Meas., vol. IM-32, no. 1, Mar. 1983.

[5] J. Doernberg, H.-S. Lee, and D. A. Hodges, "Full-speed testing of A/D converters," IEEE J. Solid-State Circuits, vol. SC-19, Dec. 1984

[6] Trial Use Standard for Digitizing Waveform Recorders, IEEE Standard 1057. New York: IEEE Press, 1989.

[7] T. M. Souders, H. K. Schoenwetter, and P. S. Hetrick. "Characterization of a sampling voltage tracker for measuring fast, repetitive signals," IEEE Trans. Instrum. Meas., vol. IM-36, Dec. 1987.

[8] C. Hagwood, G. L. Yang, and T. M. Souders, "Estimating a median using a Markov comparator," J. Appl. Probability, 1989.

[9] J. E. Freund, Mathematical Statistics, 2nd ed. Englewood Cliffs, NJ: Prentice-Hall, 1977, p. 226. 